\title{
Uncertainty of VNA S-parameter measurement due to non-ideal TMSO or LMSO calibration standards
}

\author{
U. Stumper \\ Physikalisch-Technische Bundesanstalt, Bundesallee 100, D-38116 Braunschweig, Germany
}

\begin{abstract}
For the widespread 12-term TMSO and LMSO calibration of 4-sampler vector network analyzers (VNA), the sensitivity coefficients of the S-parameters of two-ports are developed as functions of the deviations of the reflection coefficients of the one-port calibration standards and of an imperfect through or line connection. Expressions representing the deviations of the S-parameters with respect to the error terms and for the deviations of the error terms with respect to the non-ideal calibration standards are also given. It is shown that the deviations of the S-parameters become quite large particularly for high-reflective two-port test objects. If applying a broadband load (instead of using the time consuming "ideal" sliding load routine) and the VNA-internal firmware-operated calibration and evaluation routines where the reflection coefficient is set to zero, deviations may appear of some $0,1 \mathrm{~dB}$ for the attenuation and some degrees of the transmission phase angle.
\end{abstract}

\section{Introduction}

When assuming the 12-term error model with a 4-sampler vector network analyzer (VNA) and applying the widespread TMSO (or SOLT) calibration method, the one-port standards of different reflection - usually a low-reflective termination $\mathrm{M}$, a short-circuit $\mathrm{S}$ and an open circuit $\mathrm{O}$ - may be non-ideal, i.e. their reflection coefficients $M_{i}, K_{i}$, and $L_{i}$ of $\mathrm{M}, \mathrm{S}$ and $\mathrm{O}$ connected to the VNA test port numbered $i=1$ or 2 show deviations $\delta M_{i}, \delta K_{i}$, and $\delta L_{i}$ from the ideal values (or values assumed true). The through or line connection necessary for the calibration may also be nonideal. The assumption of ideal reflection values with the firmware-operated performance and evaluation of calibration and measurement of a commercial VNA then leads to deviations of the S-parameters $S_{j k}$ of the test object. To establish an uncertainty budget for the S-parameters of a test object measured after calibration, according to well known guidelines (GUM, 1995; Guidelines, 2000) it is necessary to calculate the individual uncertainty contributions - and the sensitivity coefficients - associated with the different input estimates. It is also necessary to investigate what impact the deviations of the reflection coefficients of the calibration standards have on the uncertainty of the $S_{j k}$ of test objects which include not only matched attenuators (often used as attenuation transfer standards) but also high-reflective twoports of low and high attenuation (Stumper, 2002). In the present paper, the expressions for the sensitivity coefficients are extended to the LMSO calibration method, i.e. where a line representing the characteristic line impedance of known length between the test port reference planes, is used instead of a through connection, e.g. with on-wafer measurements. The ideal through-matrix $\mathbf{T}_{\text {ideal }}=\left(\begin{array}{cc}0 & S_{12}^{T} \\ S_{21}^{T} & 0\end{array}\right)$ changes to $\mathbf{T}=\left(\begin{array}{lr}\delta S_{11}^{T} & S_{12}^{T}+\delta S_{12}^{T} \\ S_{21}^{T}+\delta S_{21}^{T} & \delta S_{22}^{T}\end{array}\right)$ for an imperfect line, where the $S_{12}^{T}, S_{21}^{T}$ are the transmission coefficients of the ideal line and the $\delta S_{11}^{T}, \delta S_{22}^{T}$ and $\delta S_{12}^{T}, \delta S_{21}^{T}$ are the reflection and transmission deviations, respectively. The general set of expressions obtained for the sensitivity coefficients is explicitly displayed, as well as the sets of expressions for the deviations of the S-parameters with respect to the error terms and for the deviations of the error terms with respect to the non-ideal calibration standards. In the calculations, the effect on the raw values e.g. of noise, non-linearity, and cross-talk is not considered (i.e. only 10 error terms are taken into account and the two additional cross-talk (or leakage) error terms are ignored in the following calculations).

\section{Measurement: dependence of the S-parameter devia- tions on error term deviations}

Ignoring the two cross-talk error terms, we consider the 10 complex error terms $E_{D}, E_{D}^{\prime}$ (directivity), $E_{S}, E_{S}^{\prime}$ (source match), $E_{R}, E_{R}^{\prime}$ (reflection tracking), $E_{T}, E_{T}^{\prime}$ (transmission tracking), and $E_{L}, E_{L}^{\prime}$ (load match). The undashed quantities denote an active test port 1 and the dashed ones an active test port 2. The $S_{j k}$ of a two-port test object are obtained from a set of four linear equations (Thumm, 1997; Gronau, 2001) given here in matrix form: 


$$
\begin{aligned}
& \left(\begin{array}{cccc}
\left(E_{S} m_{11}+E_{R}-E_{S} E_{D}\right) \cdot E_{T} & E_{R} E_{L} m_{21} & 0 & 0 \\
0 & 0 & \left(E_{S} m_{11}+E_{R}-E_{S} E_{D}\right) \cdot E_{T} & E_{R} E_{L} m_{21} \\
E_{R}^{\prime} E_{L}^{\prime} m_{12} & \left(E_{S}^{\prime} m_{22}+E_{R}^{\prime}-E_{S}^{\prime} E_{D}^{\prime}\right) \cdot E_{T}^{\prime} & 0 & 0 \\
0 & 0 & E_{R}^{\prime} E_{L}^{\prime} m_{12} & \left(E_{S}^{\prime} m_{22}+E_{R}^{\prime}-E_{S}^{\prime} E_{D}^{\prime}\right) \cdot E_{T}^{\prime}
\end{array}\right) \\
& \times\left(\begin{array}{l}
S_{11} \\
S_{12} \\
S_{21} \\
S_{22}
\end{array}\right)=\left(\begin{array}{c}
\left(m_{11}-E_{D}\right) \cdot E_{T} \\
E_{R} m_{21} \\
E_{R}^{\prime} m_{12} \\
\left(m_{22}-E_{D}^{\prime}\right) \cdot E_{T}^{\prime}
\end{array}\right) .
\end{aligned}
$$

For commercial VNAs, the tracking terms are $\left|E_{R}\right| \approx 1$, $\left|E_{R}^{\prime}\right| \approx 1,\left|E_{T}\right| \approx 1,\left|E_{T}^{\prime}\right| \approx 1$, while the magnitudes of the directivity and the source and load match are about 0,1 or smaller. The $m_{11}=m_{1} / m_{2}, m_{21}=m_{4} / m_{2}, m_{12}=m_{1}^{\prime} / m_{3}^{\prime}$, and $m_{22}=m_{4}^{\prime} / m_{3}^{\prime}$ are normalized raw values, where $m_{2}$, $m_{1}$, and $m_{4}$ are the raw (sampler) values of the incident, re- flected, and transmitted signal when test port 1 is active (cf. Fig. 1), and $m_{3}^{\prime}, m_{4}^{\prime}$, and $m_{1}^{\prime}$ the raw (sampler) values of the incident, reflected, and transmitted signal, respectively, when test port 2 is active. After rewriting the equation system and solving it with respect to the raw values, we obtain:

$$
\begin{aligned}
m_{11} & =\left\{\left[S_{11} \cdot\left(E_{R}-E_{D} E_{S}\right)+E_{D}\right] \cdot\left(1-S_{22} E_{L}\right)+S_{21} S_{12} E_{L} \cdot\left(E_{R}-E_{D} E_{S}\right)\right\} /\left\{\left(1-S_{11} E_{S}\right) \cdot\left(1-S_{22} E_{L}\right)\right. \\
& \left.-S_{21} S_{12} E_{S} E_{L}\right\}, \\
m_{21} & =S_{21} E_{T} /\left(\left(1-S_{11} E_{S}\right) \cdot\left(1-S_{22} E_{L}\right)-S_{21} S_{12} E_{S} E_{L}\right), \\
m_{12} & =S_{12} E_{T}^{\prime} /\left(\left(1-S_{22} E_{S}^{\prime}\right) \cdot\left(1-S_{11} E_{L}^{\prime}\right)-S_{12} S_{21} E_{S}^{\prime} E_{L}^{\prime}\right),
\end{aligned}
$$

$$
\begin{aligned}
& m_{22}=\left\{\left[S_{22} \cdot\left(E_{R}^{\prime}-E_{D}^{\prime} E_{S}^{\prime}\right)+E_{D}^{\prime}\right] \cdot\left(1-S_{11} E_{L}^{\prime}\right)+S_{12} S_{21} E_{L}^{\prime} \cdot\left(E_{R}^{\prime}-E_{D}^{\prime} E_{S}^{\prime}\right)\right\} /\left\{\left(1-S_{22} E_{S}^{\prime}\right) \cdot\left(1-S_{11} E_{L}^{\prime}\right)\right. \\
& \left.\quad-S_{12} S_{21} E_{S}^{\prime} E_{L}^{\prime}\right\} .
\end{aligned}
$$

We assume that the deviations $\delta S_{j k}$ are not affected by variations of the $m_{j k}$. Two total differentials, which are set to zero, can then be established considering the S-parameters $S_{11}, S_{12}, S_{21}, S_{22}$, and the undashed error terms $E_{D}, E_{S}, E_{R}$, $E_{T}, E_{L}$ (or the dashed) as variables. After execution of the differentiations we finally obtain a set of four linear equations which are uniquely solved for the four S-parameter deviations $\delta S_{11}, \delta S_{12}, \delta S_{21}$, and $\delta S_{22}$ as functions of the $10 \mathrm{de}-$ viations $\delta E_{D}, \delta E_{S}, \delta E_{R}, \delta E_{T}, \delta E_{L}, \delta E_{D}^{\prime}, \delta E_{S}^{\prime}, \delta E_{R}^{\prime}, \delta E_{T}^{\prime}$, $\delta E_{L}^{\prime}$. For $\delta S_{11}$ and $\delta S_{12}$ we obtain:

$$
\begin{aligned}
N & \delta S_{11}=-\delta E_{S} \cdot S_{11} \cdot\left[S_{11} \cdot\left(1-S_{22} E_{L}\right)+S_{21} S_{12} E_{L}\right] \cdot\left(1-S_{11} E_{L}^{\prime}\right)+\delta E_{S}^{\prime} \cdot S_{21} S_{12} \cdot\left[S_{22} \cdot\left(1-S_{11} E_{L}^{\prime}\right)+S_{12} S_{21} E_{L}^{\prime}\right] \cdot E_{L} \\
& -\delta E_{D} \cdot\left[\left(1-S_{11} E_{S}\right) \cdot\left(1-S_{22} E_{L}\right)-S_{21} S_{12} E_{S} E_{L}\right] \cdot\left(1-S_{11} E_{S}\right) \cdot\left(1-S_{11} E_{L}^{\prime}\right) / E_{R} \\
& -\delta E_{D}^{\prime} \cdot S_{21} S_{12} \cdot\left[\left(1-S_{22} E_{S}^{\prime}\right) \cdot\left(1-S_{11} E_{L}^{\prime}\right)-S_{12} S_{21} E_{S}^{\prime} E_{L}^{\prime}\right] \cdot E_{S}^{\prime} E_{L} / E_{R}^{\prime}-\delta E_{R} \cdot\left[S_{11} \cdot\left(1-S_{22} E_{L}\right)\right. \\
& \left.+S_{21} S_{12} E_{L}\right] \cdot\left(1-S_{11} E_{S}\right) \cdot\left(1-S_{11} E_{L}^{\prime}\right) / E_{R}-\delta E_{R}^{\prime} \cdot S_{21} S_{12} \cdot\left[S_{22} \cdot\left(1-S_{11} E_{L}^{\prime}\right)+S_{12} S_{21} E_{L}^{\prime}\right] \cdot E_{S}^{\prime} E_{L} / E_{R}^{\prime} \\
& +\delta E_{T} \cdot S_{21} S_{12} \cdot\left(1-S_{11} E_{L}^{\prime}\right) \cdot E_{L} / E_{T}+\delta E_{T}^{\prime} \cdot S_{12} S_{21} \cdot\left(1-S_{11} E_{L}^{\prime}\right) \cdot E_{L} / E_{T}^{\prime}-\delta E_{L} \cdot S_{21} S_{12} \\
& \cdot\left(1-S_{11} E_{L}^{\prime}\right)+\delta E_{L}^{\prime} \cdot S_{11} S_{12} S_{21} E_{L}
\end{aligned}
$$

$$
\begin{aligned}
N \cdot & \delta S_{12}=\delta E_{S} \cdot S_{11} S_{12} \cdot\left[S_{11} \cdot\left(1-S_{22} E_{L}\right)+S_{21} S_{12} E_{L}\right] \cdot E_{L}^{\prime}-\delta E_{S}^{\prime} \cdot S_{12} \cdot\left[S_{22} \cdot\left(1-S_{11} E_{L}^{\prime}\right)+S_{12} S_{21} E_{L}^{\prime}\right] \\
& \cdot\left(1-S_{22} E_{L}\right)+\delta E_{D} \cdot S_{12} \cdot\left[\left(1-S_{11} E_{S}\right) \cdot\left(1-S_{22} E_{L}\right)-S_{21} S_{12} E_{S} E_{L}\right] \cdot\left(1-S_{11} E_{S}\right) \cdot E_{L}^{\prime} / E_{R}+\delta E_{D}^{\prime} \cdot S_{12} \cdot \\
& \cdot\left[\left(1-S_{22} E_{S}^{\prime}\right) \cdot\left(1-S_{11} E_{L}^{\prime}\right)-S_{12} S_{21} E_{S}^{\prime} E_{L}^{\prime}\right] \cdot\left(1-S_{22} E_{L}\right) \cdot E_{S}^{\prime} / E_{R}^{\prime}+\delta E_{R} \cdot S_{12} \cdot\left[S_{11} \cdot\left(1-S_{22} E_{L}\right)+S_{21} S_{12} E_{L}\right] \\
& \cdot\left(1-S_{11} E_{S}\right) \cdot E_{L}^{\prime} / E_{R}+\delta E_{R}^{\prime} \cdot S_{12} \cdot\left[S_{22} \cdot\left(1-S_{11} E_{L}^{\prime}\right)+S_{12} S_{21} E_{L}^{\prime}\right] \cdot\left(1-S_{22} E_{L}\right) \cdot E_{S}^{\prime} / E_{R}^{\prime}-\delta E_{T} \cdot S_{21} \cdot \\
& \cdot S_{12}^{2} E_{L} E_{L}^{\prime} / E_{T}-\delta E_{T}^{\prime} \cdot S_{12} \cdot\left(1-S_{22} E_{L}\right) \cdot\left(1-S_{11} E_{L}^{\prime}\right) / E_{T}^{\prime}+\delta E_{L} \cdot S_{21} S_{12}^{2} E_{L}^{\prime}-\delta E_{L}^{\prime} \cdot S_{11} S_{12} \cdot\left(1-S_{22} E_{L}\right)
\end{aligned}
$$



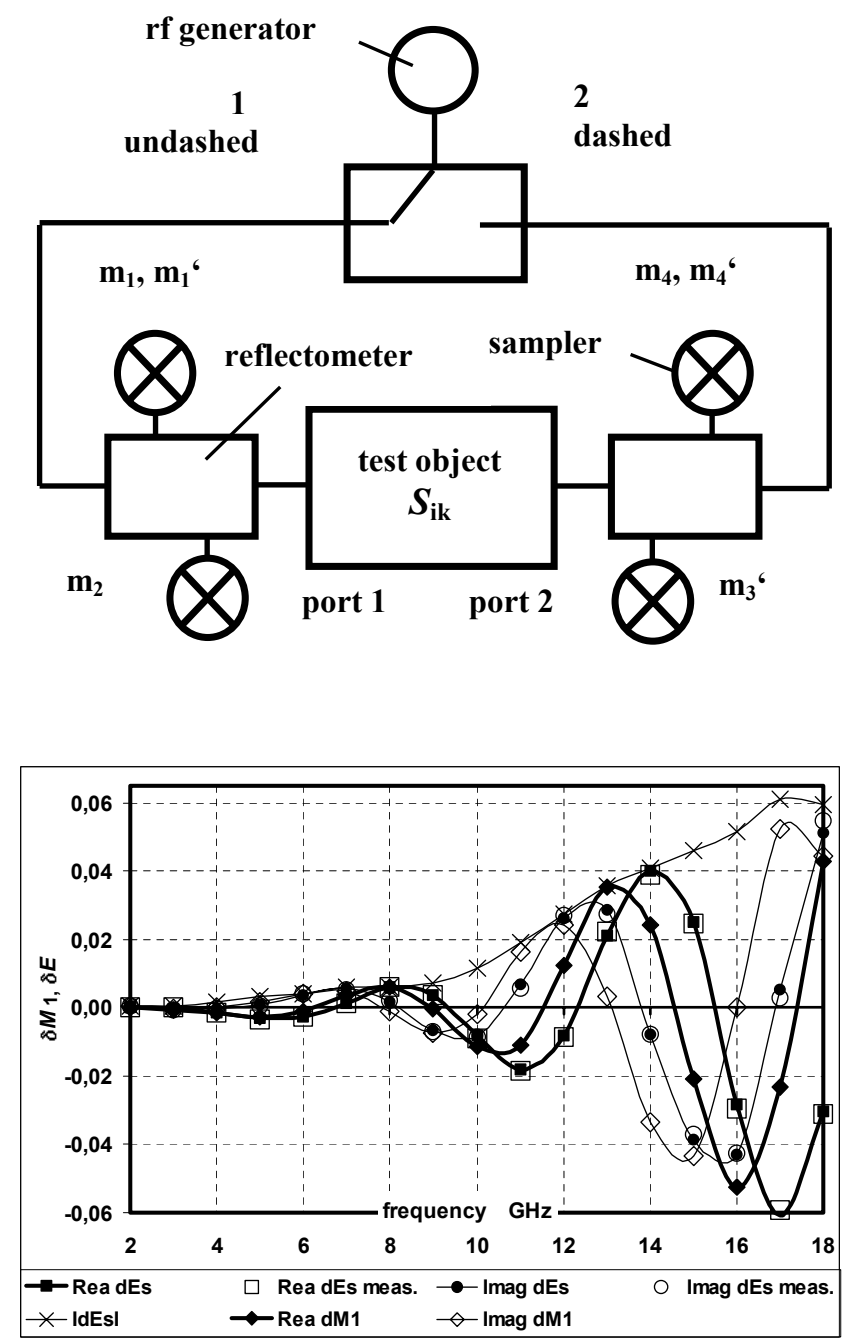

where $N=\left(1-S_{22} E_{L}\right) \cdot\left(1-S_{11} E_{L}^{\prime}\right)-S_{21} S_{12} E_{L} E_{L}^{\prime}$.

As the magnitudes of the directivity and the source and load match are small, $\delta S_{11}$ is primarily dependent on $\delta E_{S}$, $\delta E_{D}, \delta E_{R}$, and $\delta E_{L}$, and $\delta S_{12}$ primarily depends on the deviations $\delta E_{S}^{\prime}, \delta E_{T}^{\prime}$, and $\delta E_{L}^{\prime}$ of the "dashed" error terms whereas the influence of the deviations of the other error terms is smaller. Due to the symmetry of the 12-term error model, the corresponding expressions for $\delta S_{22}$ and $\delta S_{21}$ are obtained by replacing index 1 by 2 and vice versa and undashed error terms by dashed ones in Eqs. (6) and (7).

\section{Calibration: dependence of the error term deviations on reflection and through deviations}

The second step is to find out, for a full TMSO or LMSO calibration, the dependence of the error term deviations $\delta E_{D}, \ldots, \delta E_{L}^{\prime}$ on the reflection deviations $\delta M_{1}, \delta M_{2}, \delta K_{1}$, $\delta K_{2}, \delta L_{1}, \delta L_{2}$, and on the four deviations $\delta S_{11}^{T}, \delta S_{12}^{T}, \delta S_{21}^{T}$, $\delta S_{22}^{T}$ of the S-parameters of the through-connection or, alternatively, of the line inserted instead of the "through". The error terms assigned to test port 1 and test port 2 are ob-
Fig. 1. Block diagram of a 4-sampler VNA, with test port 1 active and the raw (sampler) values of the incident, reflected, and transmitted signal $m_{2}, m_{1}$, and $m_{4}$ measured, $m_{3}$ omited.

Fig. 2. Calculated (lines) and measured (signs) error term deviations $\delta E_{S}$ due to a non-ideal broadband load (of reflection Rea $\delta M_{1}$ and Imag $\left.\delta M_{1}\right)$ at port 1 for the TMSO calibration. tained from a set of ten linear equations, which can be solved with respect to the raw values $m_{j k}^{M}, m_{j k}^{K}, m_{j k}^{L}$, and $m_{j k}^{T}(T$ for "through" or, alternatively, "line"). These depend on the MSO standards of reflection coefficients $M_{1}, M_{2}, K_{1}, K_{2}$, $L_{1}, L_{2}$ and the S-parameters $S_{11}^{T}, S_{12}^{T}, S_{21}^{T}$, and $S_{22}^{T}$ of the through-connection. For active test port 1 the raw values are:

$$
\begin{aligned}
& m_{11}^{M}=\left[M_{1} \cdot\left(E_{R}-E_{S} E_{D}\right)+E_{D}\right] /\left(1-M_{1} E_{S}\right), \\
& m_{11}^{K}=\left[K_{1} \cdot\left(E_{R}-E_{S} E_{D}\right)+E_{D}\right] /\left(1-K_{1} E_{S}\right), \\
& m_{11}^{L}=\left[L_{1} \cdot\left(E_{R}-E_{S} E_{D}\right)+E_{D}\right] /\left(1-L_{1} E_{S}\right),
\end{aligned}
$$

$$
\begin{aligned}
m_{11}^{T} & =\left(\left[S_{11}^{T} \cdot\left(E_{R}-E_{D} E_{S}\right)+E_{D}\right] \cdot\left(1-S_{22}^{T} E_{L}\right)+\right. \\
& \left.+S_{21}^{T} S_{12}^{T} E_{L} \cdot\left(E_{R}-E_{D} E_{S}\right)\right) / \\
& /\left(\left(1-S_{11}^{T} E_{S}\right) \cdot\left(1-S_{22}^{T} E_{L}\right)-S_{21}^{T} S_{12}^{T} E_{S} E_{L}\right) \\
& m_{21}^{T}=S_{21}^{T} E_{T} /\left(\left(1-S_{11}^{T} E_{S}\right) \cdot\left(1-S_{22}^{T} E_{L}\right)-S_{21}^{T} S_{12}^{T} E_{S} E_{L}\right)
\end{aligned}
$$



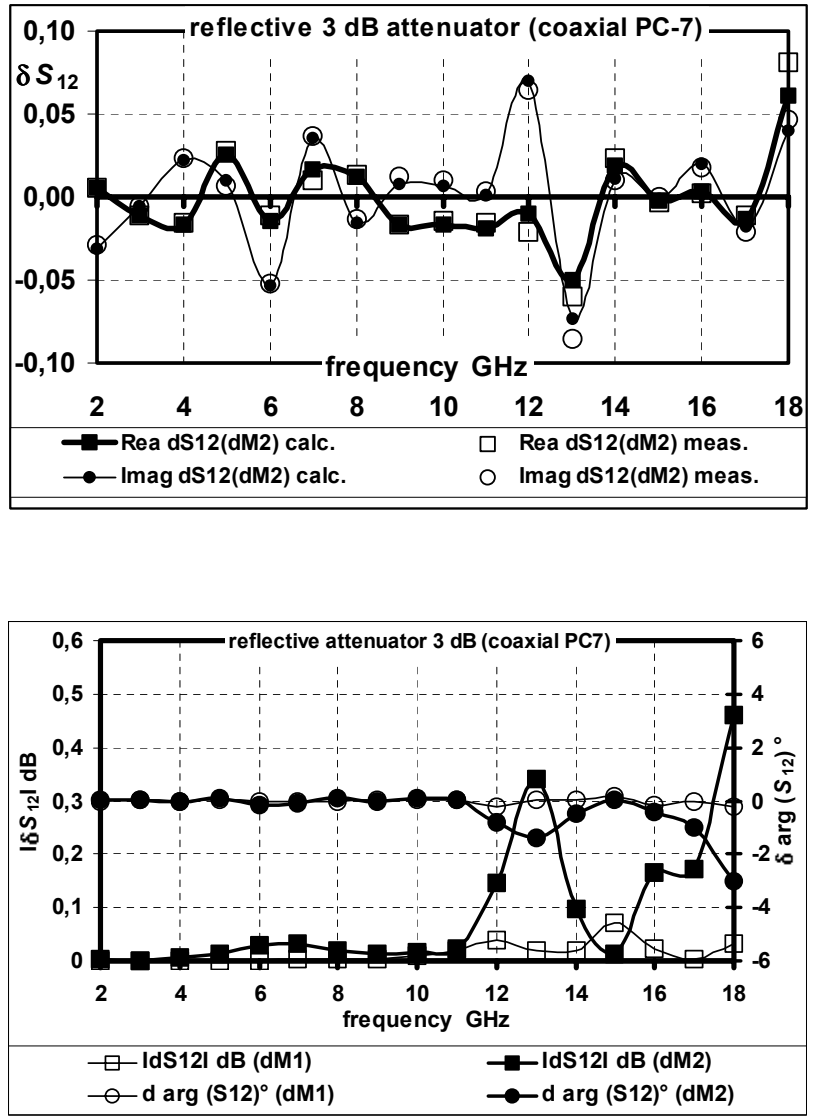

For active test port 2 we obtain a second set by replacing index 1 by 2 , and vice versa and undashed error terms by dashed ones. We assume that the deviations $\delta E, \delta E^{\prime}$ are not affected by variations of the raw values and establish 10 total differentials, namely five for active test port 1 , considering $M_{1}, K_{1}, L_{1}, S_{11}^{T}, S_{12}^{T}, S_{21}^{T}, S_{22}^{T}$ and the undashed error terms $E_{D}, E_{S}, E_{R}, E_{T}, E_{L}$ as variables and a second set of five for active test port 2 by replacing index 1 by 2 and vice versa, and undashed error terms by dashed ones. After execution of the differentiations we finally obtain a set of five linear equations assigned to the undashed error terms and a second set of five linear equations assigned to the dashed error terms. These 10 linear equations uniquely deliver the 10
Fig. 3. Comparison of calculated and measured deviations $\delta S_{12}$ for a highreflective $3 \mathrm{~dB}$ attenuator, using a mismatch instead of a sliding load at port 2 for the TMSO calibration.

Fig. 4. Calculated deviations $\left|\delta S_{12}\right|$ in $\mathrm{dB}$ and phase deviations $\delta \arg \left(S_{12}\right)$ for a high-reflective $3 \mathrm{~dB}$ attenuator using a low-reflective broadband load instead of a sliding load at port $1\left(\delta M_{1}\right)$ or port $2\left(\delta M_{2}\right)$ for the TMSO calibration (its reflection is plotted in Fig. 2). error term deviations $\delta E_{D}, \ldots, \delta E_{L}^{\prime}$ as functions of $\delta M_{1}, \delta M_{2}$, $\delta K_{1}, \delta K_{2}, \delta L_{1}, \delta L_{2}$ and $\delta S_{11}^{T}, \delta S_{12}^{T}, \delta S_{21}^{T}, \delta S_{22}^{T}$. The general expressions obtained are symmetrical with reference to the $M_{i}, K_{i}$, and $L_{i}$ which can be of arbitrary values, i.e. $\mathrm{M}, \mathrm{S}$ and $\mathrm{O}$ can be not only match, short and open circuit as used with the TMSO or LMSO calibration method but, for alternative calibration methods, can be also e.g. three offset short circuits which provide different reflection phase angles. The influence of the $\delta S_{i k}^{T}$ deviations due to a non-ideal through connection is only on transmission tracking and load match i.e. on $\delta E_{T}$ and $\delta E_{L}$, not depending on $M_{i}, K_{i}$, and $L_{i}$. We obtain for the deviation of the undashed source match:

$\delta E_{S}=-\frac{\left(1-K_{1} E_{S}\right) \cdot\left(1-L_{1} E_{S}\right)}{\left(K_{1}-M_{1}\right) \cdot\left(L_{1}-M_{1}\right)} \cdot \delta M_{1}-\frac{\left(1-M_{1} E_{S}\right) \cdot\left(1-L_{1} E_{S}\right)}{\left(M_{1}-K_{1}\right) \cdot\left(L_{1}-K_{1}\right)} \cdot \delta K_{1}-\frac{\left(1-K_{1} E_{S}\right) \cdot\left(1-M_{1} E_{S}\right)}{\left(K_{1}-L_{1}\right) \cdot\left(M_{1}-L_{1}\right)} \cdot \delta L_{1}$,

directivity:

$\delta E_{D}=-\frac{E_{R} K_{1} L_{1}}{\left(K_{1}-M_{1}\right) \cdot\left(L_{1}-M_{1}\right)} \cdot \delta M_{1}-\frac{E_{R} M_{1} L_{1}}{\left(M_{1}-K_{1}\right) \cdot\left(L_{1}-K_{1}\right)} \cdot \delta K_{1}-\frac{E_{R} K_{1} M_{1}}{\left(K_{1}-L_{1}\right) \cdot\left(M_{1}-L_{1}\right)} \cdot \delta L_{1}$,

reflection tracking:

$\delta E_{R}=-\frac{E_{R}\left[2 K_{1} L_{1} E_{S}-\left(K_{1}+L_{1}\right)\right]}{\left(K_{1}-M_{1}\right) \cdot\left(L_{1}-M_{1}\right)} \cdot \delta M_{1}-\frac{E_{R}\left[2 M_{1} L_{1} E_{S}-\left(M_{1}-L_{1}\right)\right]}{\left(M_{1}-K_{1}\right) \cdot\left(L_{1}-K_{1}\right)} \cdot \delta K_{1}-\frac{E_{R}\left[2 K_{1} M_{1} E_{S}-\left(K_{1}+M_{1}\right)\right]}{\left(K_{1}-L_{1}\right) \cdot\left(M_{1}-L_{1}\right)} \cdot \delta L_{1}$ 
transmission tracking:

$$
\begin{aligned}
\delta E_{T} & =-\frac{E_{T}\left[K_{1} L_{1} E_{S}-E_{L}\right]}{\left(K_{1}-M_{1}\right) \cdot\left(L_{1}-M_{1}\right)} \delta M_{1}-\frac{E_{T}\left[M_{1} L_{1} E_{S}-E_{L}\right]}{\left(M_{1}-K_{1}\right) \cdot\left(L_{1}-K_{1}\right)} \cdot \delta K_{1}-\frac{E_{T}\left[K_{1} M_{1} E_{S}-E_{L}\right]}{\left(K_{1}-L_{1}\right) \cdot\left(M_{1}-L_{1}\right)} \cdot \delta L_{1} \\
& -\frac{E_{T}}{S_{21}^{T}} \cdot \delta S_{21}^{T}-E_{T} E_{L} \cdot \delta S_{22}^{T},
\end{aligned}
$$

load match:

$$
\begin{aligned}
\delta E_{L} & =\frac{K_{1} L_{1}-E_{L} \cdot\left(K_{1}+L_{1}\right)+E_{L}^{2}}{\left(K_{1}-M_{1}\right) \cdot\left(L_{1}-M_{1}\right)} \cdot \delta M_{1}+\frac{M_{1} L_{1}-E_{L} \cdot\left(M_{1}+L_{1}\right)+E_{L}^{2}}{\left(M_{1}-K_{1}\right) \cdot\left(L_{1}-K_{1}\right)} \cdot \delta K_{1}+ \\
& +\frac{K_{1} M_{1}-E_{L} \cdot\left(K_{1}+M_{1}\right)+E_{L}^{2}}{\left(K_{1}-L_{1}\right) \cdot\left(M_{1}-L_{1}\right)} \cdot \delta L_{1}-\frac{1}{S_{21}^{T} S_{12}^{T}} \cdot \delta S_{11}^{T}-\frac{E_{L}}{S_{12}^{T}} \cdot \delta S_{12}^{T}-\frac{E_{L}}{S_{21}^{T}} \cdot \delta S_{21}^{T}-E_{L}^{2} \cdot \delta S_{22}^{T}
\end{aligned}
$$

The expressions simplify for the TMSO method $\left(M_{1}=\right.$ $0)$. Particularly, $\delta E_{D}=-\delta M_{1} \cdot E_{R}$ whereas the influence of $\delta K$ and $\delta L$ on directivity deviations is zero. To obtain the expressions for the dashed error term deviations $\delta E_{S}^{\prime}, \ldots, \delta E_{L}^{\prime}$, index 1 is replaced by 2 and vice versa, and $E_{S}, \ldots, E_{L}$ by $E_{S}^{\prime}, \ldots, E_{L}^{\prime}$. The influence of a given deviation $\delta M_{1}$ of a nonideal low-reflective broadband load at port 1 of the VNA on the $\delta E_{L}, \delta E_{S}$, and $\delta E_{D}$ has been tested. The values of $E_{S}$, $E_{R}$, and $E_{L}$ were obtained by either an "ideal" TMSO calibration of the VNA including a sliding load technique (i.e. $M_{1}=0$ ), a plane short circuit for $\mathrm{S}$ and a standard open circuit for O taken from the calibration kit or a "non-ideal" calibration with the low-reflective load at port 1 , while all other standards were considered as ideal. The calculated error term deviations $\delta E_{L}, \delta E_{S}$, and $\delta E_{D}$ agreed well with the differences of the values of $E_{S}, E_{R}$, and $E_{L}$, respectively, which were obtained experimentally after the "non-ideal" or the "ideal" calibration. As $\left|E_{L}\right|$ is small, $\delta E_{L} \approx \delta M_{1}$ is valid. The other deviations are also of nearly the same magnitude as $\delta M_{1}$. An example for $\delta E_{S}$ is shown in Fig. 2. The results may be used for comparison with results of the wellknown "ripple method" (Guidelines, 2000) where $\delta E_{S}$ and $\delta E_{D}$ are evaluated from the modulus of reflections measured vs. frequency of a precision air line impedance standard terminated either by a short circuit or by a low-reflective load, respectively. The applicability of the ripple method could be tested especially for thin coaxial line systems $(2,92 \mathrm{~mm}$ and $2,4 \mathrm{~mm}$ ) where reflections of an imperfect inner conductor contact at the test port become significant and therefore an increase to the $\left|\delta E_{S}\right|$ and $\left|\delta E_{D}\right|$ values may occur due to these reflections.

\section{Sensitivity coefficients: dependence of the $S$-parameters on reflection and through deviations}

By inserting Eqs. (13) to (17) - and the corresponding expressions for the dashed error term deviations - into Eqs. (6) and (7), we finally obtain 40 sensitivity coefficients of the S-parameters $S_{i k}$ of arbitrary two-port test objects with respect to the non-ideal calibration standards, represented by the deviations $\delta M_{1,2}, \delta K_{1,2}, \delta L_{1,2}, \delta S_{11}^{T}, \delta S_{12}^{T}, \delta S_{21}^{T}$, and $\delta S_{22}^{T}$. These expressions are functions of the actual values of $S_{i k}, M_{1,2}, K_{1,2}, L_{1,2}$, and the parameters $S_{21}^{T}, S_{12}^{T}$ of the through (line) connection. We obtain for $\delta S_{11}, \delta S_{12}$ and the deviations $\delta M_{1}, \delta M_{2}$ :

$\delta S_{11}(\delta M)=\frac{\left(1-S_{11} E_{L}^{\prime}\right) \cdot Z_{1 M}}{\left(K_{1}-M_{1}\right) \cdot\left(L_{1}-M_{1}\right) \cdot N} \cdot \delta M_{1}+\frac{S_{21} S_{12} E_{L} Z_{2 M}}{\left(K_{2}-M_{2}\right) \cdot\left(M_{2}-L_{2}\right) \cdot N} \cdot \delta M_{2}$,

$\delta S_{12}(\delta M)=\frac{S_{12} E_{L}^{\prime} Z_{1 M}}{\left(K_{1}-M_{1}\right) \cdot\left(M_{1}-L_{1}\right) \cdot N} \cdot \delta M_{1}+\frac{S_{12} \cdot\left(1-S_{22} E_{L}\right) \cdot Z_{2 M}}{\left(K_{2}-M_{2}\right) \cdot\left(L_{2}-M_{2}\right) \cdot N} \cdot \delta M_{2}, \quad$ where

$Z_{1 M}=\left(S_{11}-K_{1}\right) \cdot\left(S_{11}-L_{1}\right) \cdot\left(1-S_{22} E_{L}\right)-S_{21} S_{12} \cdot\left(\frac{K_{1} L_{1}}{S_{21}^{T} S_{12}^{T}}-S_{11} E_{L}\right)$,

$Z_{2 M}=S_{22} \cdot\left(1-S_{11} E_{L}^{\prime}\right)+\left(S_{12} S_{21}-S_{12}^{T} S_{21}^{T}\right) \cdot E_{L}^{\prime}+S_{11} \cdot\left[\left(K_{2}+L_{2}\right) \cdot E_{L}^{\prime}-\frac{K_{2} L_{2}}{S_{12}^{T} S_{21}^{T}}\right]$,

for the deviations $\delta K_{1}, \delta K_{2}$ :

$\delta S_{11}(\delta K)=\frac{\left(1-S_{11} E_{L}^{\prime}\right) \cdot Z_{1 K}}{\left(M_{1}-K_{1}\right) \cdot\left(L_{1}-K_{1}\right) \cdot N} \cdot \delta K_{1}+\frac{S_{21} S_{12} E_{L} Z_{2 K}}{\left(M_{2}-K_{2}\right) \cdot\left(K_{2}-L_{2}\right) \cdot N} \cdot \delta K_{2}$, 


$$
\begin{aligned}
& \delta S_{12}(\delta K)=\frac{S_{12} E_{L}^{\prime} Z_{1 K}}{\left(M_{1}-K_{1}\right) \cdot\left(K_{1}-L_{1}\right) \cdot N} \cdot \delta K_{1}+\frac{S_{12} \cdot\left(1-S_{22} E_{L}\right) \cdot Z_{2 K}}{\left(M_{2}-K_{2}\right) \cdot\left(L_{2}-K_{2}\right) \cdot N} \cdot \delta K_{2}, \quad \text { where } \\
& Z_{1 K}=\left(S_{11}-M_{1}\right) \cdot\left(S_{11}-L_{1}\right) \cdot\left(1-S_{22} E_{L}\right)-S_{21} S_{12} \cdot\left(\frac{M_{1} L_{1}}{S_{21}^{T} S_{12}^{T}}-S_{11} E_{L}\right), \\
& Z_{2 K}=S_{22} \cdot\left(1-S_{11} E_{L}^{\prime}\right)+\left(S_{12} S_{21}-S_{12}^{T} S_{21}^{T}\right) \cdot E_{L}^{\prime}+S_{11} \cdot\left[\left(M_{2}+L_{2}\right) \cdot E_{L}^{\prime}-\frac{M_{2} L_{2}}{S_{12}^{T} S_{21}^{T}}\right],
\end{aligned}
$$

for the deviations $\delta L_{1}, \delta L_{2}$ :

$$
\begin{aligned}
& \delta S_{11}(\delta L)=\frac{\left(1-S_{11} E_{L}^{\prime}\right) \cdot Z_{1 L}}{\left(K_{1}-L_{1}\right) \cdot\left(M_{1}-L_{1}\right) \cdot N} \cdot \delta L_{1}+\frac{S_{21} S_{12} E_{L} Z_{2 L}}{\left(K_{2}-L_{2}\right) \cdot\left(L_{2}-M_{2}\right) \cdot N} \cdot \delta L_{2}, \\
& \delta S_{12}(\delta L)=\frac{S_{12} E_{L}^{\prime} Z_{1 L}}{\left(K_{1}-L_{1}\right) \cdot\left(L_{1}-M_{1}\right) \cdot N} \cdot \delta L_{1}+\frac{S_{12} \cdot\left(1-S_{22} E_{L}\right) \cdot Z_{2 L}}{\left(K_{2}-L_{2}\right) \cdot\left(M_{2}-L_{2}\right) \cdot N} \cdot \delta L_{2}, \quad \text { where } \\
& Z_{1 L}=\left(S_{11}-K_{1}\right) \cdot\left(S_{11}-M_{1}\right) \cdot\left(1-S_{22} E_{L}\right)-S_{21} S_{12} \cdot\left(\frac{K_{1} M_{1}}{\left.S_{21}^{T} S_{12}^{T}-S_{11} E_{L}\right)},\right. \\
& Z_{2 L}=S_{22} \cdot\left(1-S_{11} E_{L}^{\prime}\right)+\left(S_{12} S_{21}-S_{12}^{T} S_{21}^{T}\right) \cdot E_{L}^{\prime}+S_{11} \cdot\left[\left(K_{2}+M_{2}\right) \cdot E_{L}^{\prime}-\frac{K_{2} M_{2}}{S_{12}^{T} S_{21}^{T}}\right] .
\end{aligned}
$$

The sensitivity coefficients are symmetrical with reference to the $M_{i}, K_{i}$, and $L_{i}$ which can - as well as the $S_{j k}$ - be of arbitrary values. For the through (line) deviations $\delta S_{11}^{T}, \delta S_{12}^{T}, \delta S_{21}^{T}, \delta S_{22}^{T}$ :

$$
\begin{aligned}
& \delta S_{11}\left(\delta S_{i k}^{T}\right)=\frac{S_{12} S_{21} \cdot\left(\frac{1-S_{11} E_{L}^{\prime}}{S_{21}^{T} S_{12}^{T}}-E_{L} E_{L}^{\prime}\right)}{N} \cdot \delta S_{11}^{T}-\frac{S_{11} S_{12} S_{21} E_{L} E_{L}^{\prime}}{S_{12}^{T} \cdot N} \cdot \delta S_{12}^{T}-\frac{S_{11} S_{21} S_{12} E_{L} E_{L}^{\prime}}{S_{21}^{T} \cdot N} \cdot \delta S_{21}^{T}- \\
& -\frac{S_{11} S_{12} S_{21} E_{L}}{S_{12}^{T} S_{21}^{T} \cdot N} \cdot \delta S_{22}^{T}, \\
& \delta S_{12}\left(\delta S_{i k}^{T}\right)=\frac{S_{12} E_{L}^{\prime} \cdot\left(1-S_{22} E_{L}-\frac{S_{21} S_{12}}{S_{21}^{T} S_{12}^{T}}\right)}{N} \cdot \delta S_{11}^{T}+\frac{S_{12} \cdot\left(1-S_{22} E_{L}-S_{21} S_{12} E_{L} E_{L}^{\prime}\right)}{S_{12}^{T} \cdot N} \cdot \delta S_{12}^{T}+ \\
& +\frac{S_{12} S_{11} E_{L}^{\prime} \cdot\left(1-S_{22} E_{L}\right)}{S_{21}^{T} \cdot N} \cdot \delta S_{21}^{T}+\frac{S_{12} S_{11} \cdot\left(1-S_{22} E_{L}\right)}{S_{12}^{T} S_{21}^{T} \cdot N} \cdot \delta S_{22}^{T},
\end{aligned}
$$

where $N=\left(1-S_{22} E_{L}\right) \cdot\left(1-S_{11} E_{L}^{\prime}\right)-S_{21} S_{12} E_{L} E_{L}^{\prime}$.

To obtain the sensitivity coefficients for $\delta S_{21}$ and $\delta S_{22}$, index 1 is replaced by 2 and $E_{L}$ by $E_{L}^{\prime}$ and vice versa.

\section{Experimental and calculations}

The sensitivity coefficients have been experimentally verified with a set of coaxial (PC-7) two-port test objects of (nominal) attenuation $0 \mathrm{~dB}$ to $60 \mathrm{~dB}$. A high-reflective test object was a step attenuator with three attenuation settings $(0 \mathrm{~dB}, 3 \mathrm{~dB}$, and $60 \mathrm{~dB})$. The $S_{11}$ of these objects varied with frequency $(2-18 \mathrm{GHz})$ between about $-0,6$ and $+0,6$. Low-reflective devices were of nominal $0 \mathrm{~dB}, 10 \mathrm{~dB}$, and $60 \mathrm{~dB}$ attenuation. A rather large reflection coefficient of a mismatch of VSWR 1,5 as $\mathrm{M}$ standard was used for $\delta M_{1}$ (or $\delta M_{2}$ ), while $M_{1}$ and $M_{2}$ were set to zero in the calibration routine and in the sensitivity coefficients. The calculated deviations $\delta S_{11}$ and $\delta S_{12}$ agreed well with the differences of the values of $S_{11}$ and $S_{12}$, respectively, which were obtained experimentally after calibrations using either this mismatch or an "ideal" sliding load. Results are shown in Fig. 3 for $\delta M_{2}$.

To show the influence of small values of $\delta M_{i}, \delta K_{i}$, and $\delta L_{i}$ at either port 1 or port 2 as they may occur with real calibration items from commercial calibration kits, additional 

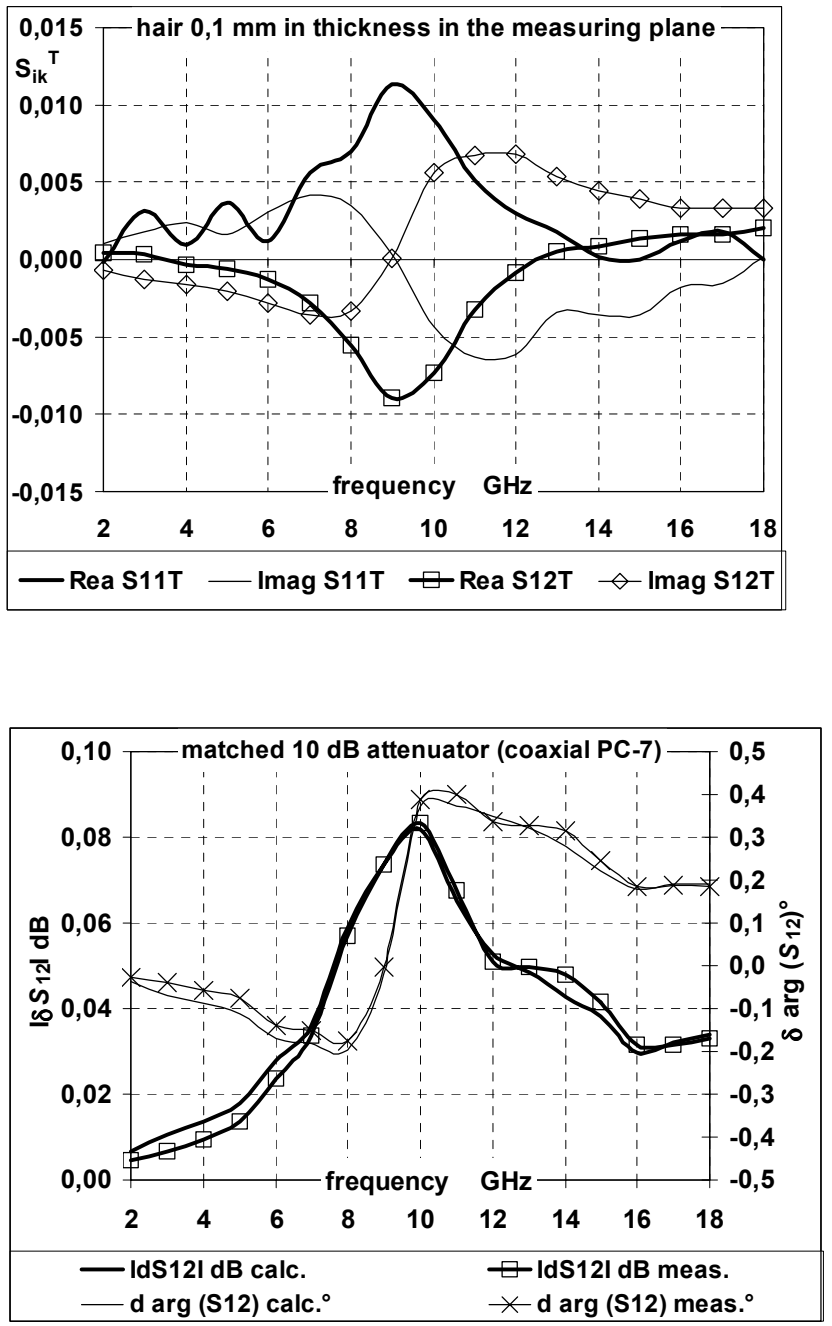

calculations have been carried out. The experimentally obtained $S_{j k}$ values of the test objects and the values for $\delta M_{1}$ or $\delta M_{2}$ of the broadband low-reflective termination (shown in Fig. 2) were used. In Fig. 4, calculated deviations of transmission $\delta S_{12}$ are shown, for the high-reflective $3 \mathrm{~dB}$ attenuator, using the low-reflective load $\left(\delta M_{2}\right)$ instead of a sliding load at port 2 for the TMSO calibration. This example shows that, if applying a broadband load of maximum reflection of 0,05 (instead of using the time consuming "ideal" sliding load routine) and the VNA-internal firmware-operated calibration and evaluation routines where the reflection coefficient of $\mathrm{M}$ is set to zero, deviations may appear of the attenuation and of the transmission phase angle in the order of $0,4 \mathrm{~dB}$ and $3^{\circ}$ respectively. For low-reflective test objects, also significant deviations of about $0,1 \mathrm{~dB}$ and $0,5^{\circ}$ may occur. The influence of a non-ideal open or short circuit with an offset length deviation of $0,01 \mathrm{~mm}$ or a short circuit loss of about $\delta K_{1}=\delta K_{2}=0,02$ at the upper coaxial band end $(18 \mathrm{GHz})$ has been found to be significantly smaller than the influence of the non-ideal broadband load (Stumper, 2003).
Fig. 5. S-parameters $S_{i k}^{T}$ of a hair $0,1 \mathrm{~mm}$ in thickness in the through connection when stretched across the connection plane of the two VNA flexible PC-7 coaxial test cables, measured with "ideal" TMSO calibration. $S_{22}^{T}$ is nearly equal to $S_{11}^{T}$ and $S_{21}^{T}$ is nearly equal to $S_{12}^{T}$.

Fig. 6. Calculated and measured deviations $\left|\delta S_{12}\right|$ in $\mathrm{dB}$ and phase deviations $\delta \arg \left(S_{12}\right)$ for a matched $10 \mathrm{~dB}$ attenuator using a non-ideal through with a hair whose S-parameters are shown in Fig. 5, instead of an ideal through, for the TMSO calibration.
The influence of a "non-ideal" through was separately investigated. To generate a non-ideal through, after having carried out a TMSO calibration with an ideal through which was then measured $\left(S_{i k \text { ideal }}^{T}\right)$, small disturbing matters of different shape and material were then inserted in the connection plane and the S-parameters $S_{i k \text { non-ideal }}^{T}$ measured. With this non-ideal through, a second calibration - now non-ideal - was then carried out. To calculate the deviations $\delta S_{i k}$ using Eqs. (30) and (31), the differences $S_{i k}^{T}=S_{i k \text { non-ideal }}^{T}-S_{i k \text { ideal }}^{T}$ were used as the $\delta S_{i k}^{T}$. For different test objects, the calculated deviations $\delta S_{i k}$ agreed well with the differences of the values of the S-parameters obtained experimentally using either the error terms of the non-ideal or of the ideal calibration. In laboratory practice, small lints may get in between the end planes of the connectors. As an example, the measured four S-parameters $S_{i k}^{T}$ (of about maximum 0,01 ) of a human hair $0,1 \mathrm{~mm}$ in thickness in the through connection are shown in Fig. 5. The deviations of modulus and phase of the transmission coefficient of a matched $10 \mathrm{~dB}$ attenuator - due to the S-parameters of this hair - are shown in Fig. 6. Even with this thin hair, the devia- 
tions are of maximum values of about $0,1 \mathrm{~dB}$ and about $0,4^{\circ}$, respectively.

\section{Conclusions}

Analytical expressions have been developed for the estimation of uncertainties in S-parameter measurement due to deviations from the ideal values of the reflection coefficients of the standards used for the 12-term LMSO or TMSO calibration and due to an imperfect line or through connection. The expressions are free of raw values and error terms except the load match. The results are experimentally verified and show that even for small deviations in the reflection values of the used standards from the "ideal" state - as assumed in the firmware evaluation - considerable deviations may be expected in the S-parameters in particular if the test objects are reflective. Even for a matched attenuator, the deviation is of the order of $0,1 \mathrm{~dB}$ if a thin hair is introduced while making the through connection.

The expressions for the sensitivity coefficients can be helpful particularly for on-wafer measurements or for coaxial lines of small diameter where the usual estimation of uncertainties including the "ripple method" yields unsatisfactory results.
Acknowledgements. The author is indebted to Thorsten Schrader for performing the verification measurements.

\section{References}

Gronau, G.: Höchstfrequenztechnik, Springer-Verlag, Berlin, Heidelberg, New York, 381-389, 2001.

Guidelines on the Evaluation of Vector Network Analysers (VNA), European Co-operation for Accreditation, Doc. EA10/12 (rev.00), 2000.

GUM: Guide to the Expression of Uncertainty in Measurement, first edition, 1993, International Organization for Standardization (Geneva, Switzerland), corrected and reprinted 1995.

Stumper, U.: Influence of calibration uncertainties on VNA Sparameter measurements, CPEM'2002 Digest, Conf. on Precision Electromagn. Meas., Ottawa, Canada, 132-133, 2002.

Stumper, U.: Influence of TMSO calibration standards uncertainties on VNA S-parameter measurements, IEEE Trans. on Instrum. Meas., 52, in print, April 2003.

Thumm, M., Wiesbeck, W., and Kern, S.: Hochfrequenzmesstechnik, Verfahren und Messsysteme, Teubner Verlag, Stuttgart, 212 213, 1997. 